\title{
The protective effects of PCPA against monocrotaline-induced pulmonary arterial hypertension are mediated through the downregulation of NFAT-1 and NF-кB
}

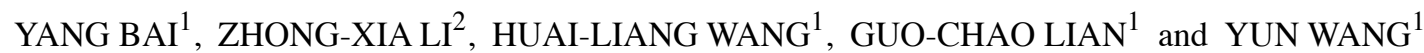 \\ ${ }^{1}$ Department of Clinical Pharmacology, College of Pharmacy, China Medical University, \\ Shenyang, Liaoning 110122; ${ }^{2}$ Department of Orthopaedic Surgery, Central Hospital Affiliated to \\ Shenyang Medical College, Shenyang, Liaoning 110000, P.R. China
}

Received May 10, 2016; Accepted May 17, 2017

DOI: $10.3892 /$ ijmm.2017.3001

\begin{abstract}
Inflammation and remodeling play a role in the pathogenesis of pulmonary arterial hypertension (PAH). Nuclear factor $-\kappa \mathrm{B}(\mathrm{NF}-\kappa \mathrm{B})$ and nuclear factor of activated T cells-1 (NFAT-1) participate in inflammation and remodeling in a number of diseases. As a tryptophan hydroxylase inhibitor, 4-chloro-DL-phenylalanine (PCPA) had been reported to exert anti-inflammatory and remodeling effects. Therefore, we hypothesized that PCPA may attenuate monocrotaline (MCT)induced PAH through the NFAT-1 and NF- $\kappa$ B signaling pathways. In order to confirm our hypothesis, we divided 68 Sprague-Dawley male rats into 4 groups as follows: the control, MCT, MCT + P1 and MCT + P2 groups. MCT was administered at a dose of $60 \mathrm{mg} / \mathrm{kg}$ once via intraperitoneal injection. PCPA was administered via intraperitoneal injection at a dose of 50 or $100 \mathrm{mg} / \mathrm{kg}$ once daily for 21 consecutive days. We then measured the hemodynamic index and morphological analysis was carried out on the lung tissues. Western blot analysis and immunohistochemistry were used to examine the levels of NFAT-1 and NF- $\kappa \mathrm{B}$ p-65. The expression levels of phosphorylated inhibitor of NF- $\kappa \mathrm{B}$ kinase (p-IKK), IKK, phosphorylated extracellular signal-regulated kinase ( $\mathrm{p}-\mathrm{ERK}$ ), ERK, intercellular adhesion molecule-1 (ICAM-1) and interleukin-6 (IL-6) were examined by western blot analysis. MCT was found to significantly induce $\mathrm{PAH}$, with inflammation and remodeling of the lung tissues. This was associatd with an increased expression of NFAT-1, p-IKK, p-ERK and
\end{abstract}

Correspondence to: Professor Yun Wang, Department of Clinical Pharmacology, College of Pharmacy, China Medical University, 77 Puhe Road, Shenyang North New Area, Shenyang, Liaoning 110122, P.R. China

E-mail: wangyun@mail.cmu.edu.cn

Key words: nuclear factor of activated T cells-1, nuclear factor- $\kappa \mathrm{B}$, 4-chloro-DL-phenylalanine, monocrotaline, pulmonary arterial hyperstension nuclear p65. PCPA significantly attenuated MCT-induced inflammation and arterial remodeling, and decreased the expression of NFAT-1, as well as that of relevant proteins of the NF- $\kappa \mathrm{B}$ signaling pathway. The above-mentioned findings suggest that the inhibitory effects of PCPA on MCT-induced inflammation and arterial remodeling are related to the downregulation of the NFAT-1 and NF- $\kappa \mathrm{B}$ signaling pathways in rats with PAH.

\section{Introduction}

Pulmonary arterial hypertension (PAH) is a complex and life-threatening disease, the characteristics of which are the continuous and evolutional rise in pulmonary vascular pressure, remodeling of the arteries, right ventricular failure and eventually, death (1). Several factors are involved in the development of the disease, including vasoconstriction, thrombosis and remodeling which have been widely recognized. Apart from these, inflammatory mechanisms also play an important role in both experimental and human pulmonary hypertension (PH) (2-4). The actions of immunity and inflammation affect multiple types of $\mathrm{PH}$, such as idiopathic $\mathrm{PAH}$ and diseases associated with $\mathrm{PAH}$, accompanied by the evaluation of circulating antibodies and pro-inflammatory cytokines, such as interleukin-1 (IL)-1 and -6 $(3,4)$.

The transcription factor, nuclear factor $-\kappa \mathrm{B}(\mathrm{NF}-\kappa \mathrm{B})$, which is made up of a homodimer or heterodimer of different members, includes p65, p50, p52, cRel and RelB (5-7). NF- $\mathrm{BB}$ is widely expressed to mediate various biological processes as a transcription factor, which activates the genes involved in inflammation, immune responses, apoptosis and cell growth (5,8-10). The most common dimers of NF- $\mathrm{B}$ are the p65 and p50 dimers. I $\kappa \mathrm{B}$, the inhibitory unit of $N F-\kappa B$, combines with $N F-\kappa B$ through its $\mathrm{C}$-terminal specific predominate ankyrin repeat sequence, and inhibits the transfer of NF- $\kappa \mathrm{B}$ to the nucleus by covering the nuclear localization sequence (NLS). In resting cells, the complexes of NF- $\kappa \mathrm{B}$ and $\mathrm{I} \kappa \mathrm{B}$ predominate in an inactive form in the cytoplasm. When the cells are stimulated by extracellular signals, I $\mathrm{KB}$ kinase (IKK) complex activation predominates I $\kappa$ B phosphorylation, and exposes NF- $\kappa$ B. 'Free' $N F-\kappa B$ then quickly shifts to the nucleus, combined with the predominance 
of a specific $\kappa \mathrm{B}$ sequence, and the induction of related gene transcription. IKK is made-up of IKK $\alpha$ and $\beta(8-10)$. p65 is an important factor in the progress of inflammation and immunity. Nuclear p65 can cause a large amount of gene transcription in reaction to inflammatory stimuli as an active form $(5,11)$.

Nuclear factor of activated T cells-1 (NFAT-1) has been reported to play a significant role in the physiopathology of pulmonary arterial smooth muscle cells (PASMCs) as an integrator of calcium signaling (12). Moreover, the transcription factor, NFAT-1, participates in chronic hypoxia induced pulmonary arterial remodeling by upregulating $\alpha$-smooth muscle actin $(\alpha$-SMA) in rats $(12,13)$. Activated NFAT participates in the process of the hypoxia-induced proliferation of human pulmonary artery smooth muscle cells (PASMCs) (14). There is also evidence to indicate that NFAT factors can interact with other nuclear transcription factors and promote the transcription of numerous inflammatory cytokines, such as activator protein 1 (AP-1), GATA binding protein 4 (GATA-4), myocyte enhancer factor-2 (MEF-2), NF- $\kappa \mathrm{B}$ p65, tumor necrosis factor (TNF) and ILs $(4,15)$.

The biosynthesis of serotonin (5-hydroxytryptamine, 5-HT) is mediated by tryptophan hydroxylase (TPH). One non-reversible inhibitor of TPH-1, 4-chloro-DL-phenylalanine (PCPA), has been shown to reduce monocrotaline (MCT)-induced inflammation and remodeling in rat lungs (16). Nevertheless, the synthesis amongst these transcriptional effector signaling pathways and their role in inflammation and remodeling have not been discussed in PAH to date, at least to the best of our knowledge. It is not known whether the inhibitory effects of PCPA on PAH are associated with the NFAT and $\mathrm{NF}-\kappa \mathrm{B}$ pathways. Therefore, in this study, we aimed to explore the mechanisms underlying the protective effects of PCPA against MCT-induced PAH, focusing on NFAT and NF- $\kappa \mathrm{B}$ in particular.

\section{Materials and methods}

Establishment of the animal models. All experiments involving rats were approved by the animal care and experimental protocols which complied with the Institutional Animal Care and Use Committee of China Medical University, Shenyang, China. All experiments abided by the guidelines of China Medical University and were approved by the local authority. Sixtyeight male Sprague-Dawley (SD) rats (weightin, 180 $10 \mathrm{~g}$ ) were obtained from the Animal Resource Centre, China Medical University (certificate no. Liaoning 034). They were separated into the following groups: i) the control group, which received the vehicle (1:4 mixture of dehydrated ethanol-normal saline) + physiological saline $(0.9 \%)$; ii) the MCT group, which received $\mathrm{MCT}+$ physiological saline; iii) the $\mathrm{MCT}+\mathrm{P} 1$ group, which received MCT + PCPA at $50 \mathrm{mg} / \mathrm{kg}$ once a day; and iv) the $\mathrm{MCT}+\mathrm{P} 2$ group, which received $\mathrm{MCT}+\mathrm{PCPA}$ at $100 \mathrm{mg} / \mathrm{kg}$ once a day.

The MCT and 2 PCPA treatment groups of rats were administered either a single dose of MCT (Sigma-Aldrich, St. Louis, MO, USA) at $60 \mathrm{mg} / \mathrm{kg}$ body weight, which was dissolved in the vehicle (1:4 mixture of dehydrated ethanolnormal saline) by intraperitoneal (i.p.) injections to induce $\mathrm{PAH}$, or the same volume of the vehicle, as previously described (2). The PCPA-effected groups of rats received PCPA (i.p.), which dissolved in physiological saline $(0.9 \%)$ continuously for 21 days once a day. Over the same period, the rats in the control and MCT group were administered an equal volume of the vehicle $(0.9 \%$ physiological saline, i.p.). All the experimental animals were fed with sufficient food and water, and were kept under a natural environment (day/night round at 50-70\% dampness and $18-22^{\circ} \mathrm{C}$ temperature). The parameters measured by us in the complete experiment were carried out in a blinded manner.

Hemodynamic measurements. On the 22nd day, all rats were narcotized with $3 \%$ sodium pentobarbital $(40 \mathrm{mg} / \mathrm{kg})$. The data of pulmonary arterial pressure (PAP) and systemic arterial pressure (SAP) were kept as records under the same factors, as previously described $(17,18)$. To measure PAP, a PV-1 catheter was inserted into the right jugular vein via the right atrium and ventricle, and was finally introduced into the pulmonary artery $(16,18)$. A polyethylene catheter (PE-50) was introduced into the right carotid artery for the measurement of SAP $(16,18)$. Hemodynamic indexes were surveyed by a pressure pickup and a polygraph system (RM6000; Nihon Kohden, Tokyo, Japan) for recording.

Pulmonary arterial morphometry. We performed histopathological observations as previously described (16). We used $4 \%$ paraform and sterile physiological saline to exsanguinate the rats. We then detached the right inferior lobe of the lungs and fixed them with $4 \%$ paraform. After embedding in paraffin, the lungs were sectioned to produce $5-\mu$ m-thick sections which were stained with hematoxylin and eosin (H\&E; ZLI-9615; ZSGB-BIO, Beijing, China). Twelve pulmonary arteries per rat were investigated using a Metamorph (Universal Imaging Corp., West Chester, PA, USA)/DP10/BX51 (Olympus, Tokyo, Japan) system in 3 rats/group (16). Percentage medial muscle thickness was measured to reflect pulmonary remodeling, which was calculated as follows: pulmonary wall thickness $(\%)=($ external diameter - internal diameter)/external diameter x100\%, as previously described (16).

Collagen and elastic fiber dyeing. The paraffin-embedded sections were stained with Orcein stain, Van Gieson stain or Victoria-ponceau's double stain (all from Shanghai Jing Ke Chemical Technology Co., Ltd., Shanghai, China) to localize elastin and collagen in lungs and pulmonary arteries.

Immunohistochemistry. We used the ultrasensitive SP and diaminobenzidine (DAB) staining kits (both from Maixin-Bio, Fuzhou, China) to stain the paraffin-embedded lung tissues and arterial sections. Primary rabbit anti-NFAT-1 (BA2799; Wuhan Boster Biological Technology, Ltd., Wuhan, China) polyclonal antibody was diluted 1:200, rabbit polyclonal anti-NF-кB p65 (sc-372; Santa Cruz Biotechnology, Inc., Santa Cruz, CA, USA) was diluted 1:300. We incubated the samples with $0.01 \mathrm{M}$ phosphate-buffered saline (PBS) in place of the primary antibody as a negative control. We then used a BX51 microscope (Olympus) to analyze the digital images. In eaach group, we observed 15 pulmonary arteries from 3 rats, the external diameter being 60-80 $\mu \mathrm{m}$. We used average optical density to calculate the NFAT-1 and p65 protein levels. 

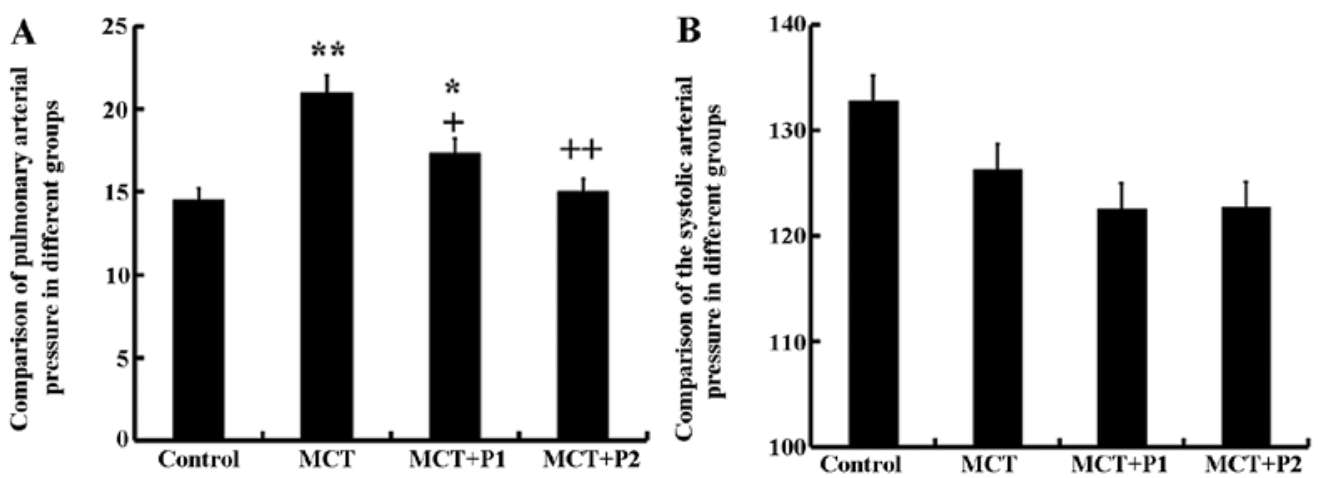

Figure 1. Comparison of haemodynamic measurements between the different groups. (A) Pulmonary arterial pressure (PAP) and (B) systolic arterial pressure were compared among the different groups. Data are shown as the means $\pm \mathrm{SD}$. ${ }^{*} \mathrm{P}<0.05$ and ${ }^{* * *} \mathrm{P}<0.01$ represent indexes compared with the control group. ${ }^{+} \mathrm{P}<0.05$, ${ }^{++} \mathrm{P}<0.01$ represent indexes compared with the monocrotaline (MCT) group. P represents 4-chloro-DL-phenylalanine (PCPA); MCT + P1 represent MCT $60 \mathrm{mg} / \mathrm{kg}$ once plus PCPA $50 \mathrm{mg} / \mathrm{kg}$ body weight a day for 21 days; MCT + P2 represent MCT $60 \mathrm{mg} / \mathrm{kg}$ once plus PCPA $100 \mathrm{mg} / \mathrm{kg}$ body weight a day for 21 days.

Preparation of total protein. To obtain total protein, the inferior lobe of the left lungs and pulmonary arteries were homogenized using a polytron homogenizer (Kinematica, Lucerne, Switzerland). We then utilized the Heraeus Sepatech to separate the homogenate at $4^{\circ} \mathrm{C}$ and $15,000 \mathrm{x}$ g for $20 \mathrm{~min}$. The supernatant was collected and storing it at $-70^{\circ} \mathrm{C}$ for further analysis. The total proteins were determined by the Bradford method.

Preparation of nuclear and cytosolic protein. Nuclear and cytosolic proteins were prepared as previously described (19). The fresh samples were homogenized and the homogenate was then collected using a nuclear protein extraction kit (Beyotime Institute of Biotechnology, Haimen, China). The extract methods of nuclear fractions and cytosolic fractions were according to the manufacturer's instructions. We stored the aliquots at $-70^{\circ} \mathrm{C}$ until analysis. Using BCA (Beyotime Institute of Biotechnology) method determined the nuclear and cytosolic protein concentration.

Western blot analysis. The same amount of protein was segregated through SDS-PAGE and electro-transferred onto polyvinylidene difluoride (PVDF) membranes, as previously described (17). The PVDF membranes were incubated with 5\% non-fat dry milk, 1X TBS and $0.05 \%$ Tween-20 for $2 \mathrm{~h}$ at room temperature. The primary antibodies, rabbit polyclonal anti-NFAT-1 antibody (1:400, BA2799; Wuhan Boster Biological Technology, Ltd.), rabbit polyclonal anti-extracellular signal-regulated kinase (ERK)1/2 antibody (1:500, sc-292838), mouse monoclonal anti-p-ERK1/2 (1:500, sc-81492), rabbit polyclonal anti-IKK $\alpha$ antibody (1:500, sc-7218), rabbit polyclonal antip-IKK $\alpha$ antibody (1:600, sc-101706) and rabbit polyclonal anti-NF-кB p65 antibody (1:400, sc-372) (all from Santa Cruz Biotechnology, Inc.), goat polyclonal anti-intercellular adhesion molecule-1 (ICAM-1) antibody (1:200, zs-1511; Bioworld Technology, Inc., St. Louis Park, MN, USA), goat polyclonal anti-IL-6 antibody (1:300, sc-1265), mouse monoclonal anti- $\alpha$-tubulin antibody $(1: 2,000, \mathrm{sc} 8035)$ and mouse polyclonal anti- $\beta$-actin antibody $(1: 2,000, \mathrm{sc}-47778)$ (all from Santa Cruz Biotechnology, Inc.) were incubated with the membranes at $4^{\circ} \mathrm{C}$ overnight. The following day, the PVDF membranes were incubated with the corresponding horseradish peroxidase (HRP)-conjugated secondary antibodies at room temperature for $2 \mathrm{~h}$. Subsequently, using the super ECL plus (Thermo Fisher Scientific, Inc., Waltham, MA, USA) were observed the immunoreactive bands. Densitometry was used to quantify the relative protein expression using Quantity One software (Bio-Rad Laboratories, Inc., Hercules, CA, USA).

Statistical analysis. The data are presented as the means \pm standard deviation (SD). SPSS version 16.0 sofware (SPSS, Inc., Chicago, IL, USA) was used to carry out all the statistic analyses. One-way analysis of variance (ANOVA) with Fisher's least significant difference (LSD) or Dunnett's T3 test were used for statistical comparative analysis. A value of $\mathrm{P}<0.05$ was considered to indicate a statistically significant difference.

\section{Results}

Influence of PCPA on MCT-induced haemodynamics and morphological changes in rats. The rats in all groups were fed for 21 days. In the MCT group, the mean PAP was significantly increased (21.06 $\pm 3.4 \mathrm{mmHg}, \mathrm{P}<0.01$ vs. control group). PCPA significantly inhibited the mean PAP; in the MCT + P1 group PAP decreased to $17.4 \pm 2.5 \mathrm{mmHg}(\mathrm{P}<0.05)$ and in the $\mathrm{MCT}+\mathrm{P} 2$ group, it decreased to $15.1 \pm 3.1 \mathrm{mmHg}(\mathrm{P}<0.01)$ compared with the MCT group. However, the values of SAP in the 4 groups exhibited no significant differences (Fig. 1).

The muscularization of lung tissue was examined under a light microscope. In the MCT group, the thickness of the arterial wall was significantly increased $(49.7 \pm 9.2 \%, \mathrm{P}<0.01$ vs. control $19.1 \pm 7.7 \%)$. PCPA inhibited this thickness in a dose-dependent manner in the MCT + P1 group $(34.3 \pm 8.2 \%$, $\mathrm{P}<0.01$ vs. MCT $)$ and $\mathrm{MCT}+\mathrm{P} 2$ group $(28.1 \pm 10.7 \%, \mathrm{P}<0.01$ vs. MCT) (Fig. 2).

Evaluation of elastin and collagen. Fig. 3 shows double staining in elastin and collagen in the lungs. We observed that the collagen was conspicuously diffused and increased in the MCT group, and the elastic fibers were also disrupted and increased in the MCT group. PCPA inhibited collagen deposition and decreased the structural destruction of the lungs in a 

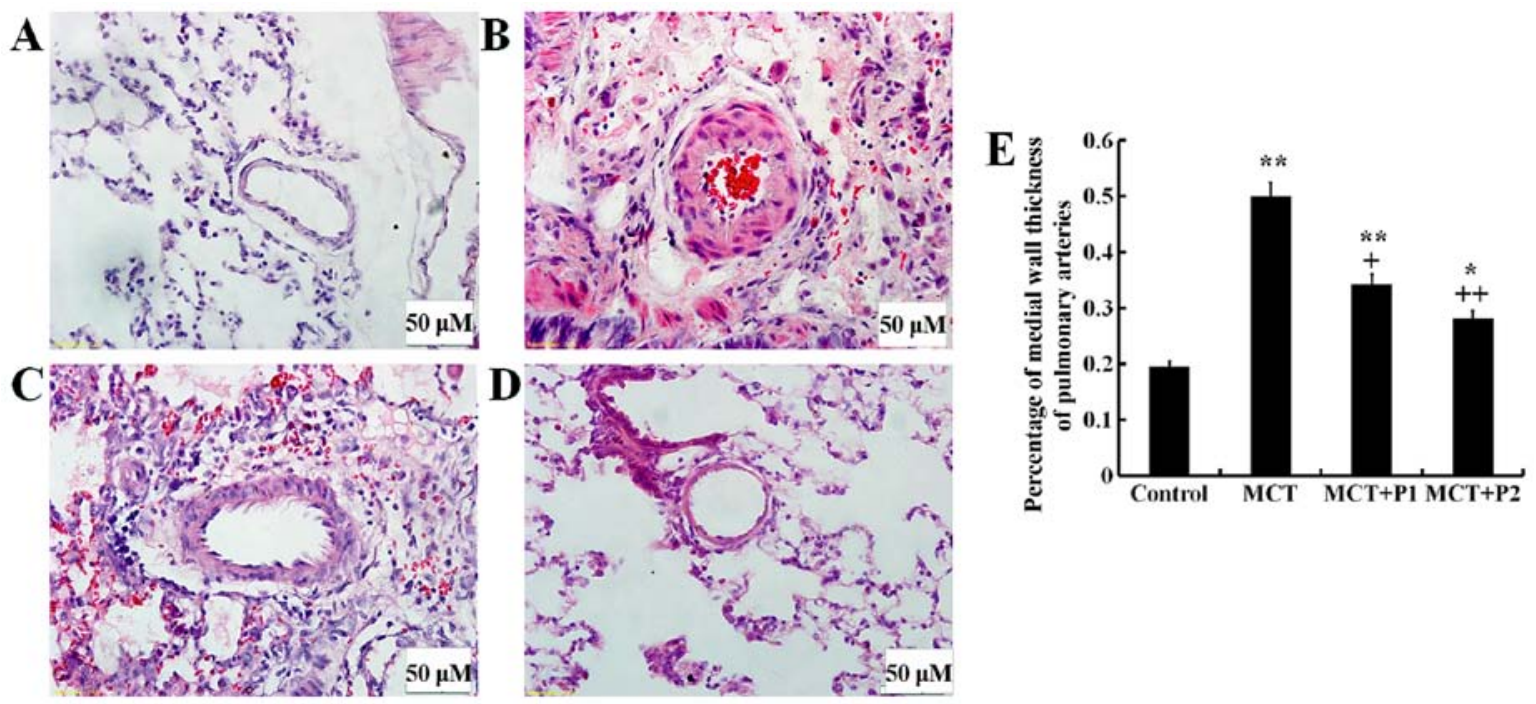

Figure 2. Results of the effects 4-chloro-DL-phenylalanine (PCPA) on pulmonary arterial wall thickness ratio are compared. (A) Control group, (B) monocrotaline (MCT) group, (C) MCT + P1 group (PCPA at $50 \mathrm{mg} / \mathrm{kg}$ body weight) and (D) MCT + P2 group (PCPA at $100 \mathrm{mg} / \mathrm{kg}$ body weight); (E) percentage of medical wall thickness of pulmonary arteries. Original magnification, $\mathrm{x} 400$; scale bars, $50 \mu \mathrm{m}$. Data are shown as the means $\pm \mathrm{SD}\left(\mathrm{n}=3\right.$ rats). ${ }^{*} \mathrm{P}<0.05$, ${ }^{* *} \mathrm{P}<0.01$ represent indexes compared with the control group. ${ }^{+} \mathrm{P}<0.05$ and ${ }^{++} \mathrm{P}<0.01$ represent indexes compared with the MCT group.
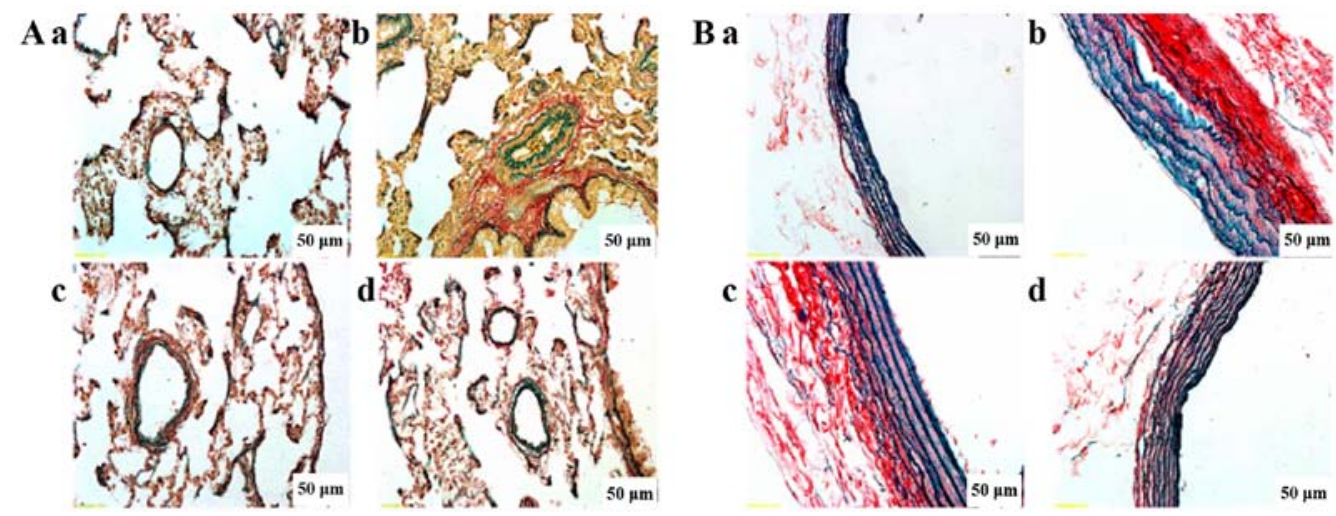

Figure 3. Comparison of elastin and collagen deposition between the different groups. (A) Double staining in elastin and collagen in lungs. (B) Double staining in elastin and collagen in main pulmonary arteries. (a) Control group, (b) monocrotaline (MCT) group, (c) MCT + P1 group (PCPA at $50 \mathrm{mg} / \mathrm{kg}$ body weight) and (d) MCT + P2 group (PCPA at $100 \mathrm{mg} / \mathrm{kg}$ body weight). Bluish green represents elastin and the red represents collagen. Original magnification, $\mathrm{x} 400$; scale bars, $50 \mu \mathrm{m}$.

dose-dependent manner. In particular, the high dose of PCPA markedly inhibited elastin and collagen hyperplasia and maintained the integrity of the arterial structure simultaneously.

Influence of PCPA on NFAT-1 expression. Immunohistochemistry revealed a stronger expression of NFAT-1 in the group with MCT-induced PAH using anti-NFAT-1 antibodies compared with the controls. PCPA (50 mg/kg/day) had little influence on NFAT-1 protein in rats. However, treatment with PCPA at $100 \mathrm{mg} / \mathrm{kg} /$ day led to marked decrease in the expression of NFAT-1 compared with the MCT group, in which NFAT-1 expression was increased (Fig. 4).

The results of western blot analysis revealed that the rats in the MCT group had significantly elevated protein expression levels of NFAT-1 compared with the control group rats $(1.03 \pm 0.01$ vs. $0.59 \pm 0.04$, respectively, $\mathrm{P}<0.01)$. PCPA $(50 \mathrm{mg} / \mathrm{kg})$ suppressed NFAT-1 protein expression $(0.78 \pm 0.04, \mathrm{P}<0.05 \mathrm{vs}$. MCT $)$. However, $\mathrm{PCPA}$ at $100 \mathrm{mg} / \mathrm{kg}$ decreased NFAT-1 protein expression, which was increased by MCT more significantly $(0.67 \pm 0.05, \mathrm{P}<0.01$ vs. MCT) (Fig. 5).

Evaluation of $N F-\kappa B$ p 65 . Immunohistochemistry revealed a stronger p65 expression in the rat lungs of the MCT group using anti-p65 antibodies compared with the control group. Treatment with PCPA at $50 \mathrm{mg} / \mathrm{kg} / \mathrm{day}$ led to a slight, yet significant decrease in $\mathrm{p} 65$ protein expression. The results revealed that treatment with PCPA at $100 \mathrm{mg} / \mathrm{kg} /$ day even more significantly reduced the increase in $\mathrm{p} 65$ expression induced by MCT (Fig. 6).

Western blot analysis revealed that the expression of $\mathrm{p} 65$ protein was markedly increased in the nuclear protein isolated from the lungs of the rats in the MCT group compared with those of the control group ( $\mathrm{P}<0.01 \mathrm{vs}$. control). PCPA at $50 \mathrm{mg}$ / $\mathrm{kg}$ suppressed MCT-induced nuclear p65 expression, but without statistical significance ( $>>0.05$ vs. MCT). However, PCPA at $100 \mathrm{mg} / \mathrm{kg}$ markedly attenuated the expression of 

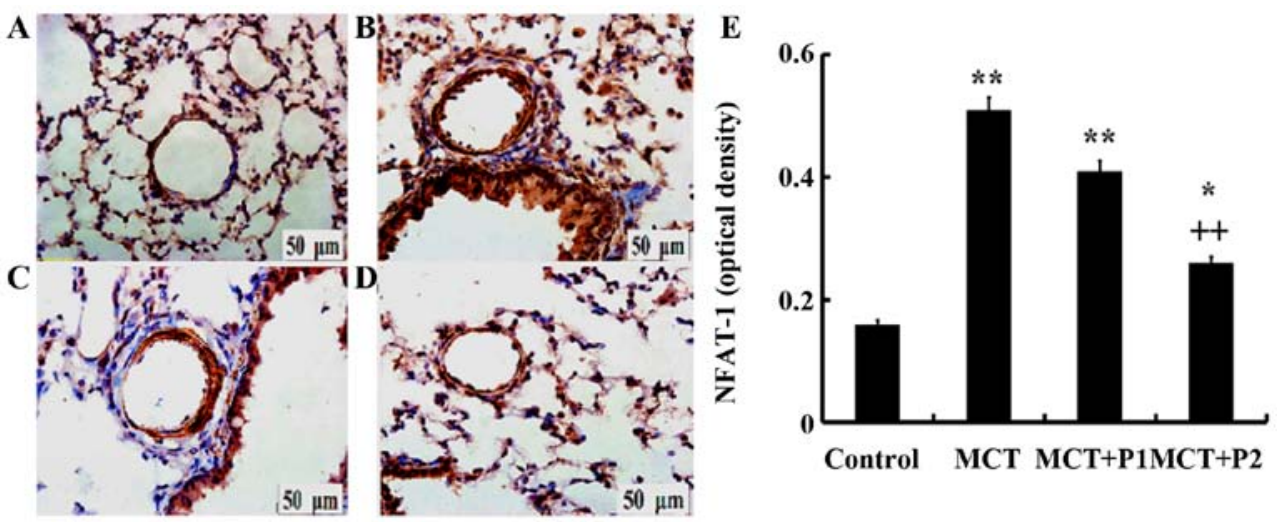

Figure 4. Immunohistochemical determination of nuclear factor of activated T cells-1 (NFAT-1) expression in lung vessels. (A) Control group, (B) monocrotaline (MCT) group, (C) MCT + P1 group (PCPA at $50 \mathrm{mg} / \mathrm{kg}$ body weight), (D) MCT + P2 group (PCPA at $100 \mathrm{mg} / \mathrm{kg}$ body weight) and (E) average optical density of NFAT-1. Original magnification, $\mathrm{x} 400$; scale bars, $50 \mu \mathrm{m}$. Data are shown as the means $\pm \mathrm{SD}\left(\mathrm{n}=3\right.$ rats). ${ }^{*} \mathrm{P}<0.05,{ }^{* *} \mathrm{P}<0.01$ represent indexes compared with the control group. ${ }^{++} \mathrm{P}<0.01$ represents indexes compared with the MCT group.

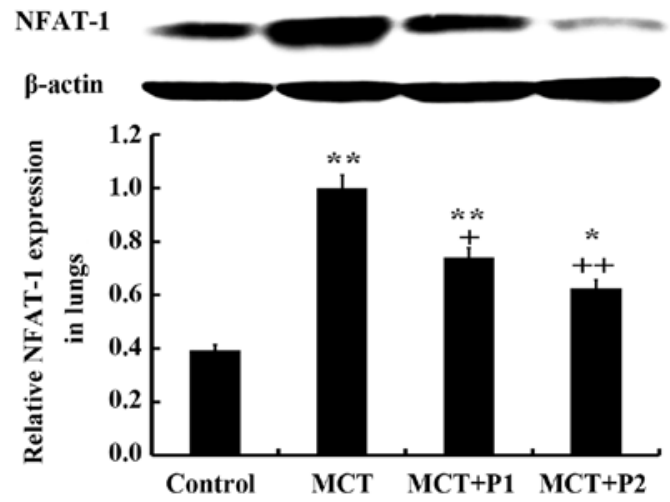

Figure 5. Western blot analysis of the expression of nuclear factor of activated T cells-1 (NFAT-1) in lung tissues of rats with monocrotaline (MCT)-induced pulmonary arterial hypertension (PAH). Comparisons between the NFAT-1 and the control group, MCT-induced pulmonary arterial hypertension (PAH) group and 4-chloro-DL-phenylalanine (PCPA) treatment groups were made. Data are shown as the means $\pm \mathrm{SD}\left(\mathrm{n}=5\right.$ rats). ${ }^{*} \mathrm{P}<0.05,{ }^{* * *} \mathrm{P}<0.01$ represent indexes compared with the control group. ${ }^{+} \mathrm{P}<0.05$ and ${ }^{++} \mathrm{P}<0.01$ represent indexes compared with the MCT group. P represents 4-chloro-DL-phenylalanine (PCPA); $\mathrm{MCT}+\mathrm{P} 1$ represent MCT $60 \mathrm{mg} / \mathrm{kg}$ once plus PCPA $50 \mathrm{mg} / \mathrm{kg}$ body weight a day for 21 days; MCT + P2 represent MCT $60 \mathrm{mg} / \mathrm{kg}$ once plus PCPA $100 \mathrm{mg} /$ $\mathrm{kg}$ body weight a day for 21 days.
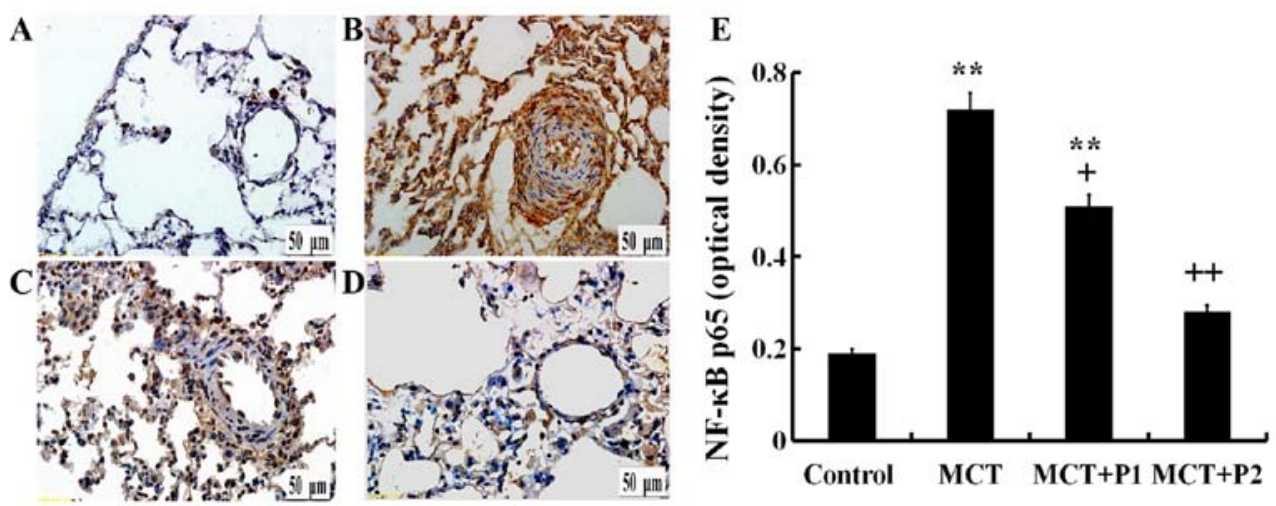

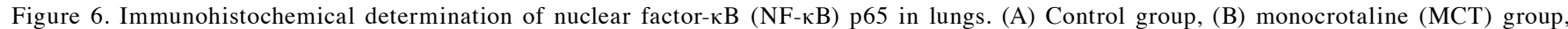
(C) MCT + P1 group, (D) MCT + P2 group and (E) average optical density of p65. Original magnification, x400; scale bars, $50 \mu \mathrm{m}$. Data are shown as the means $\pm \mathrm{SD}\left(\mathrm{n}=3\right.$ rats). ${ }^{* *} \mathrm{P}<0.01$ represents indexes compared with the control group. ${ }^{+} \mathrm{P}<0.05$ and ${ }^{++} \mathrm{P}<0.01$ represent indexes compared with the MCT group. P represents 4-chloro-DL-phenylalanine (PCPA); MCT + P1 represent MCT $60 \mathrm{mg} / \mathrm{kg}$ once plus PCPA $50 \mathrm{mg} / \mathrm{kg}$ body weight a day for 21 days; MCT + P2 represent MCT $60 \mathrm{mg} / \mathrm{kg}$ once plus PCPA $100 \mathrm{mg} / \mathrm{kg}$ body weight a day for 21 days.

nuclear p65 induced by MCT ( $<<0.01$ vs. MCT) (Fig. 7). No significant difference was observed in the cytoplasmic expression of p65 among the 4 groups.
Effect of PCPA on ERK, p-ERK, IKK and p-IKK expression. Western blot analysis revealed an increased level of phosphorylated ERK in the lungs of the MCT group compared 

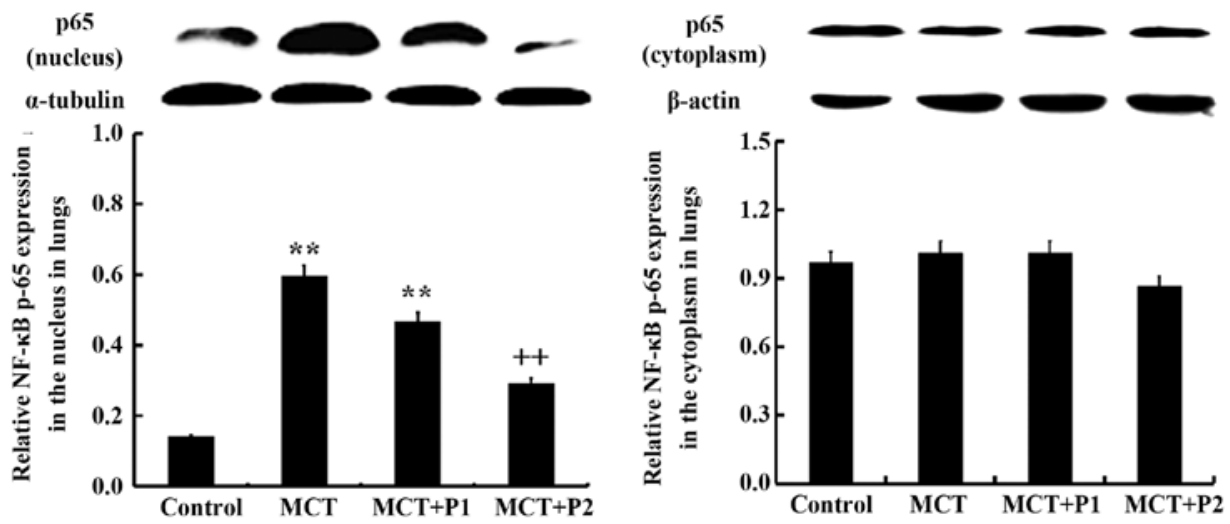

Figure 7. Comparison of nuclear factor- $\mathrm{kB}(\mathrm{NF}-\mathrm{\kappa B}) \mathrm{p} 65$ expression in nucleus and cytoplasm in rat lungs by western blot analysis. Comparisons between the NF- $\mathrm{KB}$ p65 among different groups, control, monocrotaline (MCT)-induced pulmonary arterial hypertension (PAH) and 4-chloro-DL-phenylalanine (PCPA) treatment groups were made. Data are shown as the means $\pm \mathrm{SD}\left(\mathrm{n}=5\right.$ rats). ${ }^{* *} \mathrm{P}<0.01$ represents indexes compared with the control group. ${ }^{++} \mathrm{P}<0.01$ represents indexes compared with the MCT group. P represents 4-chloro-DL-phenylalanine (PCPA); MCT + P1 represent MCT $60 \mathrm{mg} / \mathrm{kg}$ once plus PCPA $50 \mathrm{mg} / \mathrm{kg}$ body weight a day for 21 days; MCT + P2 represent MCT $60 \mathrm{mg} / \mathrm{kg}$ once plus PCPA $100 \mathrm{mg} / \mathrm{kg}$ body weight a day for 21 days.

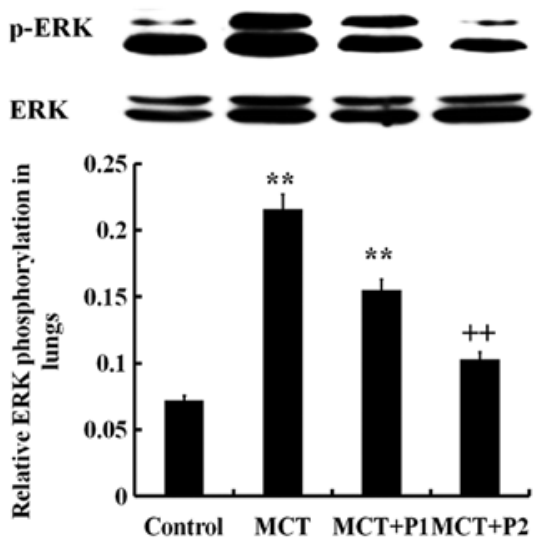

Figure 8. Western blot analysis of the phosphorylation levels of extracellular signal-regulated kinase (ERK) in lungs. Comparisons of ERK phosphorylation among different groups, control, monocrotaline (MCT)-induced pulmonary arterial hypertension (PAH) and 4-chloro-DL-phenylalanine (PCPA) treatment groups, were made. Data are shown as the means $\pm \mathrm{SD}$ ( $\mathrm{n}=5$ rats). ${ }^{* * *} \mathrm{P}<0.01$ represents indexes compared with the control group. ${ }^{++} \mathrm{P}<0.01$ represents indexes compared with the MCT group. P represents 4-chloro-DLphenylalanine (PCPA); MCT + P1 represent MCT $60 \mathrm{mg} / \mathrm{kg}$ once plus PCPA $50 \mathrm{mg} / \mathrm{kg}$ body weight a day for 21 days; MCT + P2 represent MCT $60 \mathrm{mg} / \mathrm{kg}$ once plus PCPA $100 \mathrm{mg} / \mathrm{kg}$ body weight a day for 21 days.

with the control group ( $\mathrm{P}<0.01$; Fig. 8). PCPA decreased the ERK phosphorylation level induced by MCT, particularly in the MCT + P2 group ( $\mathrm{P}<0.01$ vs. MCT) (Fig. 8). The IKK phosphorylation level in the MCT group was significantly increased $(\mathrm{P}<0.01$ vs. control). Treatment with PCPA at $100 \mathrm{mg} / \mathrm{kg} /$ day notably decreased IKK phosphorylation in the lungs $(\mathrm{P}<0.01$ vs. MCT) (Fig. 9). These results indicated that PCPA inhibited the MCT-induced activation of ERK and IKK in the lungs.

Influence of PCPA on ICAM-1 and IL-6 expression. Fig. 10 shows that ICAM-1 and IL-6 expression was markedly increased by MCT compared to the control. The expression of ICAM-1 was elevated from $0.45 \pm 0.02$ in the control group to $1.61 \pm 0.26$ in the MCT group $(\mathrm{P}<0.01)$. PCPA at $50 \mathrm{mg} / \mathrm{kg} / \mathrm{day}$ decreased ICAM-1 expression to $1.24 \pm 0.12(\mathrm{P}>0.05)$ compared with the MCT group, and PCPA at $100 \mathrm{mg} / \mathrm{kg} /$ day reduced

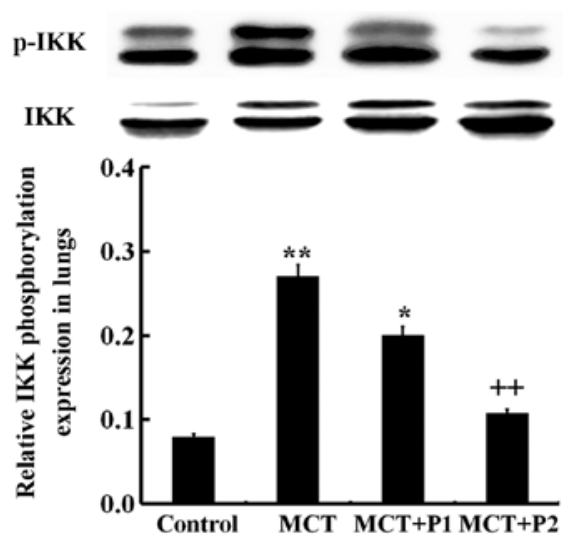

Figure 9. Western blot analysis of the phosphorylation levels of IкB kinase (IKK) in lungs. IKK phosphorylation in the different groups, control, monocrotaline (MCT)-induced pulmonary arterial hypertension (PAH) and the 50 and $100 \mathrm{mg} / \mathrm{kg}$ 4-chloro-DL-phenylalanine (PCPA) treatment groups was examined. Data are shown as the means $\pm \mathrm{SD}$ ( $\mathrm{n}=5$ rats). ${ }^{*} \mathrm{P}<0.05$ and ${ }^{* *} \mathrm{P}<0.01$ represent indexes compared with the control group. ${ }^{++} \mathrm{P}<0.01$ represents indexes compared with the MCT group. P represents 4-chloro-DLphenylalanine (PCPA); MCT + P1 represent MCT $60 \mathrm{mg} / \mathrm{kg}$ once plus PCPA $50 \mathrm{mg} / \mathrm{kg}$ body weight a day for 21 days; MCT + P2 represent MCT $60 \mathrm{mg} / \mathrm{kg}$ once plus PCPA $100 \mathrm{mg} / \mathrm{kg}$ body weight a day for 21 days.

ICAM-1 expression to $0.98 \pm 0.15(\mathrm{P}<0.05)$. Similarly, the expression of IL-6 was markedly increased by MCT, from $0.61 \pm 0.04$ in the control group to $1.42 \pm 0.21(\mathrm{P}<0.01)$ in the MCT group. PCPA at $50 \mathrm{mg} / \mathrm{kg} /$ day decreased IL-6 expression to $0.96 \pm 0.05(\mathrm{P}<0.05)$ compared with the MCT group, and PCPA at $100 \mathrm{mg} / \mathrm{kg} /$ day reduce IL-6 expression to $0.69 \pm 0.15(\mathrm{P}<0.01)$ (Fig. 10). Thus, PCPA, particularly at a high dose reduced the expression of IL-6 and ICAM-1.

\section{Discussion}

In this study, we demonstrated that the expression levels of NFAT-1, nuclear NF-кB p65, phosphorylated ERK, IKK, IL-6 and ICAM-1 were markedly elevated by MCT in the lungs. PCPA markedly reduced the expression of NFAT- 1 and proteins related to the NF- $\mathrm{KB}$ signaling pathway, and thus inhibited lung inflammation and remodeling. This is a demonstration 

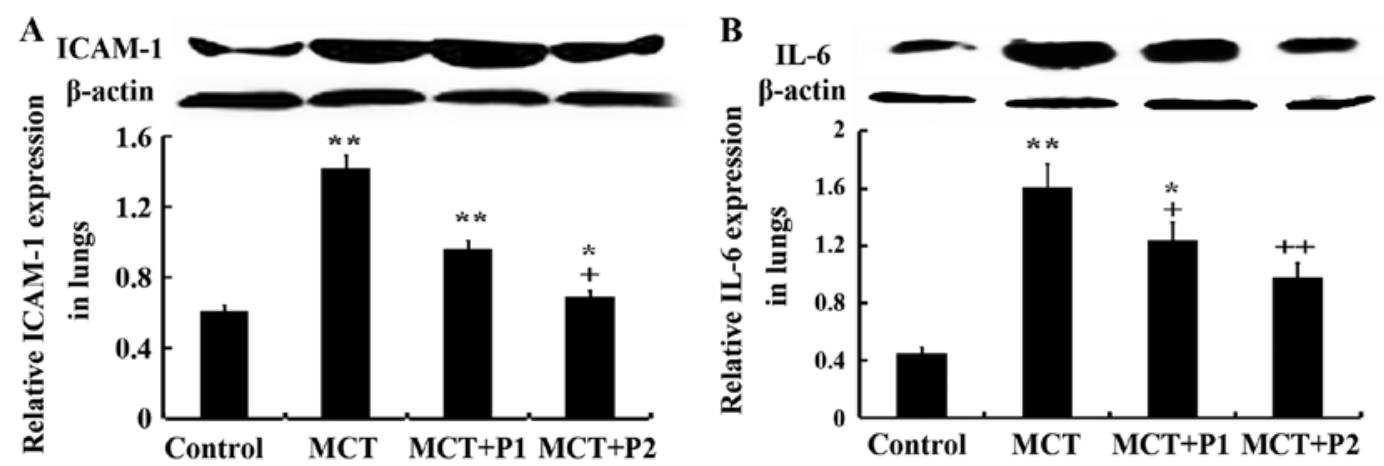

Figure 10. Western blot analysis of the expression of (A) intercellular adhesion molecule-1 (ICAM-1) and (B) interleukin-6 (IL-6) in rat lungs. Comparisons of ICAM-1 and IL-6 expression in different groups, control group, monocrotaline (MCT)-induced pulmonary arterial hypertension (PAH) group and 4-chloro-DL-phenylalanine (PCPA) treatment groups were made. Data are shown as the means $\pm \mathrm{SD}\left(\mathrm{n}=5\right.$ rats). ${ }^{*} \mathrm{P}<0.05$ and ${ }^{* *} \mathrm{P}<0.01$ represent indexes compared with the control group. ${ }^{+} \mathrm{P}<0.05$ and ${ }^{++} \mathrm{P}<0.01$ represents indexes compared with the MCT group.

of the inter-dependence between NFAT and NF- $\kappa \mathrm{B}$ signaling in mediating lung tissue inflammation, which has an effect on MCT-induced PAH; the 5-HT signaling pathways may be involved in this process.

The occurrence of PAH is a complex pathophysiological process, and its pathogenesis is not yet completely clear. There is evidence to indicate that the synthesis of 5-HT may influence the pathological process of PAH. Our laboratory previously has proved that PCPA inhibited 5-HT, TPH-1, serotonin transporter (SERT), serotonin receptor and associated serotonin signaling pathways (16). The PCPA exerted protective effects against PCPA by attenuating MCT-induced lung inflammation and remodeling, and this was related to the reduction of SERT, TPH-1, matrix metalloproteinases (MMPs), tissue inhibitors of metalloproteinases (TIMPs) and some inflammatory factors (16). Evidence indicates that PCPA inhibits inflammation in lungs by suppressing TPH in allergic airway inflammation models (20).

Fluoxetine, is an inhibitor of SERT, and its inhibitory effects on pulmonary vascular remodeling in MCT-induced PAH are mediated through the downregulation of the ERK, Akt and RhoA/ROCK signaling pathways (17). In addition, fluoxetine suppresses lung tissue inflammation $(17,18)$. Ketanserin, a 5-HT2A receptor antagonist, had been shown to modestly suppress inflammation and eosinophil infiltration in allergic airway inflammation models (21). These findings demonstrate that 5-HT is involved in the processes of remodeling and lung inflammation in PAH. The phenomenon can be decreased by inhibiting the 5-HT upstream and downstream signaling pathways.

NFAT, as a transcription factor, promotes the transcription of a number of inflammatory cytokines, such as ILs and TNF, and can activate T and B cells (22). Bonnet et al (23) demonstrated that in PAH, including scleroderma-associated PAH and idiopathic PAH (IPAH), NFATc2 is upregulate and activated in some circulating inflammatory cells. A recent study found that following treatment with 5-HT, calcineurin and NFAT pathway activation occurred in PASMCs, which was related to dosage (24). In addition, following treatment with sidenafil, an inhibitor of PDE5, the serotonin-induced activation of calcineurin/NFATc2 signaling pathway was suppressed, which suggested that one of the major targets of sildenafil was the calcineurin/NFAT cascade in the pulmonary system (24). In the present study, the expression of NFAT-1 was markedly increased in the MCT group, which was inhibited by PCPA in a dose-dependent manner. Consistent with previous results, inference with PCPA may regulate NFAT-1 by inhibiting serotonin.

$\mathrm{NF}-\kappa \mathrm{B}$ is involved in inflammation, immunoreactions and various other biological processes. Several factors can activate $\mathrm{NF}-\kappa \mathrm{B}$, including inflammatory cytokines, growth factors or chemokines, such as transforming growth factor- $\beta$ (TGF- $\beta$ ), serotonin and connective tissue growth factor (CTGF). A $\mathrm{NF}-\kappa \mathrm{B}$ homodimer or a heterodimer is formed by two types of subunits, a class of subunit p65 and another type of subunit p50 and p52. p65, a particularly curcial subunit, can mediate the processes of inflammation and tumor formation (5). $\mathrm{NF}-\kappa \mathrm{B}$ can be activated by the multi-subunit IKK composed of two catalytic subunits, IKK $\alpha$ and $\beta$, which responds to various cellular stimuli, including bacterial or viral antigens, cytokines, growth factors and mitogens (25). In unstimulated cells, $\mathrm{NF}-\kappa \mathrm{B}$ is usually found in the cytoplasm and binds to inhibitory proteins $\mathrm{I} \kappa \mathrm{Bs}(25,26)$. Upon activation, I $\mathrm{I} B$ is phosphorylated by IKK, and thus $\mathrm{I} \kappa \mathrm{B} \alpha$ is degraded and $\mathrm{NF}-\kappa \mathrm{B}$ is released and then translocates to the nucleus $(27,28)$. Rapidly, $\mathrm{I} \kappa \mathrm{B} \alpha$ can be synthesized by the NF- $\kappa \mathrm{B}$-mediated expression of its gene (29,30). In addition, as a pivotal mediator in signal transduction, $\mathrm{NF}-\kappa \mathrm{B}$ is involved in the effecter phase of inflammation when responding to multiple inflammatory cytokines, e.g., IL-1 and TNF (31). In addition to promoting the activation of a wide range of cytokines, activated $\mathrm{NF}-\kappa \mathrm{B}$ also promotes the expression of ICAM-1, -2 and other adhesion molecules and nitric oxide syntheses (NOS) by activating endothelial cells (32-34).

In the present study, we found that the expression levels of nuclear p65, IKK phosphorylation, ICAM-1 and IL-6 were significantly increased by MCT in the lungs. PCPA markedly suppressed the NF- $\kappa$ B p65 nuclear translocation, inhibited IKK phosphorylation and inflammatory cytokine production. The above-mentioned results indicated that PCPA suppressed 5-HT, mediating the NF- $\mathrm{NB}$ signaling pathway. These inflammatory mediators or cytokines can stimulate the systemic inflammatory response syndrome, increasing the damage to the lungs.

There is synergy between NF- $\kappa \mathrm{B}$ and NFAT, which can facilitate transcriptional activation of each other in cardiomyocytes (26). Liu et al (26) demonstrated that a complex 
was formed through the direct interaction of NFAT with p65, promoting $\mathrm{NF}-\kappa \mathrm{B}$ nuclear translocation induced by IKK $\beta$ and NFAT nuclear localization enhanced by p65-RHD. In accordance with the results from other studies, our study showed that NFAT-1 promoted IKK phosphorylation and dissociation through the IKK-NF- $\mathrm{B}$ signaling pathway, and p65 nuclear translocation. Activated NF- $\kappa B$ can promote the production of large amounts of IL- 6 and other cellular factor, and can then activate endothelial cells to produce ICAM-1.

During the progression of PAH, factors which can regulate the stability of p65 are of importance. It has been demonstrated that ERK plays a crucial role in transferring inflammatory information from the extracellular environment to the cytoplasm or nucleus, which can modulate inflammatory responses $(35,36)$. The ERK signaling pathway can regulate PASMCs exposed to 5-HT during mitosis (37). The ERK and NFAT-3 signal pathways and hyperphosphate-induced response have a marked effect on the development of cardiac hypertrophy (38). In our previous study, MCT-induced vascular remodeling in lungs, which was caused by the crosstalk among SERT, RhoA/ROCK and ERK signaling pathway, was suppressed by fluoxetine (17). Targeting NF- $\mathrm{B}$ can secondarily suppress the NFAT signaling pathway, and can thus be considered as a original therapeutic method in putting into use cardiac hypertrophy (26). Our present results demonstrate that PCPA suppressed the expression and activation of the phosphorylation ERK in a dose-dependent manner, indicating that PCPA attenuates inflammation and remodeling in PAH by mediating the NFAT-1 and IKK-NF- $\kappa$ B signaling pathways.

In conclusion, our study demonstrates that PCPA exerts a protection effect on MCT-induced inflammation and remodeling in lungs, which is related to the NFAT and NF- $\kappa \mathrm{B}$ signaling pathways. The detailed mechanisms however, require further investigation.

\section{Acknowledgements}

This study was supported by the Science Research Project of the Education Department of Liaoning Province (LK201640), the National Natural Science Foundation of China (nos. 81273511 and 81503058) and the Natural Science Foundation of Liaoning Province (no. 2014021065).

\section{References}

1. Lai YC, Potoka KC, Champion HC, Mora AL and Gladwin MT: Pulmonary arterial hypertension: the clinical syndrome. Circ Res 115: 115-130, 2014

2. Chaumais MC, Ranchoux B, Montani D, Dorfmüller P, Tu L, Lecerf F, Raymond N, Guignabert C, Price L, Simonneau G, et al: $\mathrm{N}$-acetylcysteine improves established monocrotaline-induced pulmonary hypertension in rats. Respir Res 15: 65, 2014.

3. Rabinovitch M, Guignabert $C$, Humbert $M$ and Nicolls MR: Inflammation and immunity in the pathogenesis of pulmonary arterial hypertension. Circ Res 115: 165-175, 2014.

4. El Chami H and Hassoun PM: Immune and inflammatory mechanisms in pulmonary arterial hypertension. Prog Cardiovasc Dis 55: 218-228, 2012

5. Liu J, Sha M, Wang Q, Ma Y, Geng X, Gao Y, Feng L, Shen Y and Shen Y: Small ubiquitin-related modifier 2/3 interacts with p65 and stabilizes it in the cytoplasm in HBV-associated hepatocellular carcinoma. BMC Cancer 15: 675, 2015.

6. DiDonato JA, Mercurio F and Karin M: NF- $\kappa$ B and the link between inflammation and cancer. Immunol Rev 246: 379-400, 2012 .
7. Hoesel B and Schmid JA: The complexity of NF- $\kappa B$ signaling in inflammation and cancer. Mol Cancer 12: 86, 2013.

8. Vallabhapurapu S and Karin M: Regulation and function of NF-kappaB transcription factors in the immune system. Annu Rev Immunol 27: 693-733, 2009.

9. Hayden MS and Ghosh S: NF- $\kappa$ B, the first quarter-century: remarkable progress and outstanding questions. Genes Dev 26: 203-234, 2012.

10. Zhang $\mathrm{H}$ and Sun SC: NF- $\kappa$ B in inflammation and renal diseases. Cell Biosci 5: 63, 2015.

11. Gilmore TD and Wolenski FS: NF- $\mathrm{BB}$ : where did it come from and why? Immunol Rev 246: 14-35, 2012.

12. Parpaite T, Cardouat G, Mauroux M, Gillibert-Duplantier J, Robillard P, Quignard JF, Marthan R, Savineau JP and Ducret T: Effect of hypoxia on TRPV1 and TRPV4 channels in rat pulmonary arterial smooth muscle cells. Pflugers Arch 468: 111-130, 2016.

13. de Frutos S, Spangler R, Alò D and Bosc LV: NFATc3 mediates chronic hypoxia-induced pulmonary arterial remodeling with alpha-actin up-regulation. J Biol Chem 282: 15081-15089, 2007.

14. Wang C, Li JF, Zhao L, Liu J, Wan J, Wang YX, Wang J and Wang C: Inhibition of SOC/Ca ${ }^{2+} / \mathrm{NFAT}$ pathway is involved in the anti-proliferative effect of sildenafil on pulmonary artery smooth muscle cells. Respir Res 10: 123, 2009.

15. Hogan PG, Chen L, Nardone J and Rao A: Transcriptional regulation by calcium, calcineurin, and NFAT. Genes Dev 17: 2205-2232, 2003.

16. Bai Y, Wang HM, Liu M, Wang Y, Lian GC, Zhang XH, Kang J and Wang HL: 4-Chloro-DL-phenylalanine protects against monocrotaline induced pulmonary vascular remodeling and lung inflammation. Int J Mol Med 33: 373-382, 2014.

17. Wang HM, Wang Y, Liu M, Bai Y, Zhang XH, Sun YX and Wang HL: Fluoxetine inhibits monocrotaline-induced pulmonary arterial remodeling involved in inhibition of RhoA-Rho kinase and Akt signalling pathways in rats. Can J Physiol Pharmacol 90: 1506-1515, 2012.

18. Li XQ, Wang HM, Yang CG, Zhang XH, Han DD and Wang HL: Fluoxetine inhibited extracellular matrix of pulmonary artery and inflammation of lungs in monocrotaline-treated rats. Acta Pharmacol Sin 32: 217-222, 2011.

19. Yang G, Abate A, George AG, Weng YH and Dennery PA: Maturational differences in lung NF-kappaB activation and their role in tolerance to hyperoxia. J Clin Invest 114: 669-678, 2004.

20. Dürk T, Duerschmied D, Müller T, Grimm M, Reuter S, Vieira RP, Ayata K, Cicko S, Sorichter S, Walther DJ, et al: Production of serotonin by tryptophan hydroxylase 1 and release via platelets contribute to allergic airway inflammation. Am J Respir Crit Care Med 187: 476-485, 2013.

21. De Bie JJ, Henricks PA, Cruikshank WW, Hofman G, Jonker EH, Nijkamp FP and Van Oosterhout AJ: Modulation of airway hyperresponsiveness and eosinophilia by selective histamine and 5-HT receptor antagonists in a mouse model of allergic asthma. Br J Pharmacol 124: 857-864, 1998.

22. Macian F: NFAT proteins: key regulators of T-cell development and function. Nat Rev Immunol 5: 472-484, 2005.

23. Bonnet S, Rochefort G, Sutendra G, Archer SL, Haromy A, Webster L, Hashimoto K, Bonnet SN and Michelakis ED: The nuclear factor of activated $\mathrm{T}$ cells in pulmonary arterial hypertension can be therapeutically targeted. Proc Natl Acad Sci USA 104: 11418-11423, 2007.

24. Li M, Liu Y, Sun X, Li Z, Liu Y, Fang P, He P, Shi H, Xie M, Wang X, et al: Sildenafil inhibits calcineurin/NFATc2-mediated cyclin A expression in pulmonary artery smooth muscle cells. Life Sci 89: 644-649, 2011.

25. Kang HJ, Hong SH, Kang KH, Park C and Choi YH: Anti-inflammatory effects of Hwang-Heuk-San, a traditional Korean herbal formulation, on lipopolysaccharide-stimulated murine macrophages. BMC Complement Altern Med 15: 447, 2015

26. Liu Q, Chen Y, Auger-Messier M and Molkentin JD: Interaction between NFKB and NFAT coordinates cardiac hypertrophy and pathological remodeling. Circ Res 110: 1077-1086, 2012.

27. Karin $M$ and Ben-Neriah Y: Phosphorylation meets ubiquitination: the control of NF-[kappa]B activity. Annu Rev Immunol 18: 621-663, 2000.

28. Hayden MS and Ghosh S: Signaling to NF-kappaB. Genes Dev 18: 2195-2224, 2004

29. Sun SC, Ganchi PA, Ballard DW and Greene WC: NF-kappa B controls expression of inhibitor I kappa B alpha: evidence for an inducible autoregulatory pathway. Science 259: 1912-1915, 1993. 
30. Peng B, Ling J, Lee AJ, Wang Z, Chang Z, Jin W, Kang Y, Zhang R, Shim D, Wang H, et al: Defective feedback regulation of NF-kappaB underlies Sjogren's syndrome in mice with mutated kappaB enhancers of the IkappaBalpha promoter. Proc Natl Acad Sci USA 107: 15193-15198, 2010.

31. Tak PP and Firestein GS: NF-kappaB: a key role in inflammatory diseases. J Clin Invest 107: 7-11, 2001.

32. Tamura R, Chen Y, Shinozaki M, Arao K, Wang L, Tang W, Hirano S, Ogura H, Mitsui T, Taketani S, et al: Eudesmane-type sesquiterpene lactones inhibit multiple steps in the NF- $\mathrm{BB}$ signaling pathway induced by inflammatory cytokines. Bioorg Med Chem Lett 22: 207-211, 2012.

33. Foulds S, Galustian C, Mansfield AO and Schachter M: Transcription factor NF kappa B expression and postsurgical organ dysfunction. Ann Surg 233: 70-78, 2001.

34. Bhatia M, Brady M, Shokuhi S, Christmas S, Neoptolemos JP and Slavin J: Inflammatory mediators in acute pancreatitis. J Pathol 190: 117-125, 2000.
35. Kim KN, Ko SC, Ye BR, Kim MS, Kim J, Ko EY, Cho SH, Kim D, Heo SJ and Jung WK: 5-Bromo-2-hydroxy-4methyl-benzaldehyde inhibited LPS-induced production of pro-inflammatory mediators through the inactivation of ERK, p38, and NF- $\kappa$ B pathways in RAW 264.7 macrophages. Chem Biol Interact 258: 108-114, 2016.

36. Xu G, Feng L, Song P, Xu F, Li A, Wang Y, Shen Y, Wu X, Luo Q, Wu X, et al: Isomeranzin suppresses inflammation by inhibiting M1 macrophage polarization through the NF- $\mathrm{BB}$ and ERK pathway. Int Immunopharmacol 38: 175-185, 2016.

37. Liu Y, Suzuki YJ, Day RM and Fanburg BL: Rho kinase-induced nuclear translocation of ERK1/ERK2 in smooth muscle cell mitogenesis caused by serotonin. Circ Res 95: 579-586, 2004

38. Liu YL, Huang CC, Chang CC, Chou CY, Lin SY, Wang IK, Hsieh DJ, Jong GP, Huang CY and Wang CM: Hyperphosphate-induced myocardial hypertrophy through the GATA-4/NFAT-3 signaling pathway is attenuated by ERK inhibitor treatment. Cardiorenal Med 5: 79-88, 2015. 